\title{
Análisis del comportamiento temporal de la biomasa en Pastos del Departamen- to de Olancho en el contexto del cambio global - Fase I
}

José David Cáceres

\section{Resumen}

Según la guía de buenas prácticas del Panel Intergubernamental de Expertos en Cambio Climático (IPCC), las técnicas de teledetección son adecuadas para la estimación de los sumideros de carbono a partir de la estimación de la biomasa. En este trabajo, concebido en dos fases, se utilizará una serie temporal de imágenes Landsat para estimar la cantidad de biomasa del pasto en el Departamento de Olancho. En la fase I, se ha delimitado la zona de estudio derivada del análisis de información territorial suministrada por el Sistema Nacional de Información Territorial (SINIT), así como análisis visual de imágenes Landsat e imágenes de alta resolución. Se obtuvo un área de cobertura de pastos de 670,749.79 ha lo que representa aproximadamente un $28 \%$ de la extensión total del Departamento. Se elaboraron protocolos de radiometría de campo para la estandarización y fiabilidad de la información recopilada por personal del Laboratorio de Radiometría del Departamento de Ciencia y Tecnologías de la Información Geográfica.

Palabras Clave: Landsat; pastos; radiometría; biomasa; stocks de carbono

\section{Abstract}

According to the guide of good practices Intergovernmental Panel on Climate Change (IPCC), remote sensing techniques are suitable for the estimation of carbon sinks from the biomass estimate. In this work, conceived in two phases, a time series of Landsat images will be used to estimate the amount of grass biomass in the Department of Olancho. In phase I, the study area has been derived from the analysis of spatial information provided by the National Land Information System (SINIT) and visual analysis of Landsat images and high resolution images. We obtained an area of grass cover in the department of Olancho $670,749.79$ ha representing approximately $28 \%$ of the total area of the Department. Measurement protocols 
were developed for field radiometry standardization and reliable survey information collected by staff of the Radiometry Laboratory of the Department of Science and Geographic Information Technologies.

Keywords: Landsat; pastures; radiometry; biomass carbon stocks

José David Cáceres, (jcaceres@unah.edu.hn), Departamento de Ciencia y Tecnologías de la Información Geográfica,Facultad de Ciencias Espaciales ,Universidad Nacional Autónoma de Honduras 


\section{Introducción}

Las masas forestales juegan un papel fundamental en el ciclo del carbono, fijándolo de la atmósfera y almacenándolo en su biomasa, y constituyen, por ende, uno de los más importantes sumideros de carbono. Ante los compromisos suscritos por los países, incluido Honduras, para la mitigación del cambio climático en la Convención Marco de Las Naciones Unidas sobre el Cambio Climático (CMNUCC), la cuantificación del balance de carbono se presenta como uno de los principales desafíos al permitir considerar la fijación de carbono como un objetivo más de la gestión forestal. Esto conlleva la estimación del carbono almacenado en la biomasa, así como el seguimiento de la evolución de estos stocks a diferentes escalas temporales.

La estimación de los stocks de carbono en zonas heterogéneas, como sabanas o potreros, utilizando sensores remotos, representa desafíos únicos debido a la presencia de dos estratos de vegetación con un comportamiento muy diferenciado, el pasto y los árboles.(Todd et al. 1998)

Este estudio se basa en el uso de índices espectrales, derivados de imágenes Landsat, para estimar biomasa en una zona de pastos en el momento de máxima actividad vegetativa, que corresponde con la época de primavera (marzo-mayo). A partir de una serie temporal de mediciones de campo obtenidas de un sitio con características similares a la zona de estudio propuesta e imágenes Landsat se generará un modelo empírico para la estimación de biomasa en pastos en el Departamento de Olancho.

En enero de 2010 surge lo que se llama Plan de Nación y Visión de País la cual declara 16 principios orientadores del desarrollo el cual el sexto está alineado con la educación y desarrollo humano para la generación de oportunidades. En su objetivo el No.3 "Una Honduras productiva, generadora de oportunidades y empleo, que aprovecha de manera sostenible sus recursos y reduce la vulnerabilidad ambiental.", nos enmarca en la importancia de la gestión sostenible de nuestros recursos naturales, esta visión de país lleva de la mano un proceso basado en el desarrollo territorial el cual define a Honduras como un territorio ambiental, cultural y territorialmente diverso debido a estas características se establece el Modelo de Desarrollo Regional el cual establece en La Ley de Visión de País y Plan de Nación 16 Regiones de Desarrollo, delimitadas por las cuencas principales del país.

El lineamento estratégico número 11 de este documento nos resalta la importancia de contar con información que permita dirigir los esfuerzos hacia la 
adaptación y mitigación al cambio climático, siendo la estimación de los stocks de carbono, a través de la biomasa, un pilar fundamental en la consecución de este objetivo. Dentro de las prioridades de investigación de la UNAH, este proyecto se encuentra enmarcado en la Prioridad No. 11: Cambio Climático y Vulnerabilidad.

El objetivo general de este estudio consiste en construir un modelo estadístico para la estimación de biomasa en pastos en la zona oriental del país. Para la consecución de este objetivo se ha dividido el estudio en dos fases y han planteado varios objetivos específicos, los cuáles se mencionan a continuación:

- Elaborar protocolos para el uso del laboratorio y la toma de información espectral de especies de pastos en campo utilizando el radiómetro. (Fase I)

- Utilizar la capa de Uso del Suelo elaborada por el Sistema Nacional de Información Territorial (SINIT), para las zonas con cobertura de pastos (sistema agrosilvopastoril). (Fase I)

- Elaborar un mapa de la zona de estudio del proyecto. (Fase I)

- Realizar campañas de mediciones periódicas del comportamiento espectral de los pastos. (Fase II)

- Comparar las firmas espectrales recolectadas con el comportamiento espectral de las zonas identificadas para validar la presencia de pastos. (Fase II)

- Construir un modelo de regresión lineal para la estimación de biomasa en pastos a partir de índices espectrales. (Fase II)

\section{Metodología}

La metodología descrita en este apartado corresponde al desarrollo de la Fase I del presente proyecto.

\section{- Diseño Metodológico}

Para la consecución de los objetivos planteados, se definió un esquema metodológico en 3 etapas (Figura 1). En primer lugar, se procedió a la delimitación del área de estudio. La definición del área se realizó utilizando como base la cartografía generada por el Sistema Nacional de Información Territorial (SINIT), identificando y seleccionando la zona correspondiente a la cobertura Agropecua- 
rio, se realizó una depuración realizando un análisis visual de las coberturas de la imagen satelital de referencia del sensor TM, empleando una combinación en falso color mejorado (4-5-3), así como información de apoyo como ser imágenes de alta resolución disponibles a través de la plataforma Google Earth. Luego se sobrepuso la capa de red vial, se realizó un buffer de 6 metros sobre esta capa y se procedió a eliminar de la capa de Uso Agropecuario todas las áreas que cayeran dentro del área de influencia creada para la capa de red vial, obteniéndose así el área de estudio final. La segunda etapa corresponde a la composición del Mapa del área de estudio utilizando el software especializado. En la última etapa, aunque en paralelo a las dos anteriores, se procedió a la elaboración de un protocolo de medición de radiometría de campo utilizando como referencia la experiencia personal y los protocolos utilizados por el Laboratorio de Espectro-radiometría y Teledetección Ambiental del Consejo Superior de Investigaciones Científicas (CSIC) - Centro de Ciencias Humanas y Sociales (CCHS).

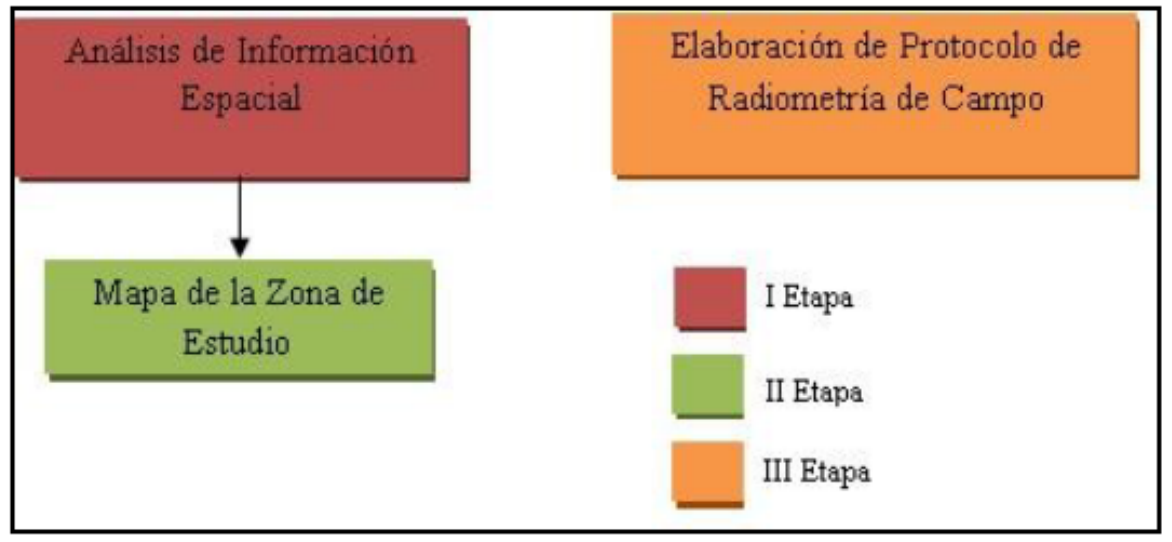

Figura 1. Diagrama de las Etapas de la Me

\section{Antecedentes}

En la última década, la teledetección ha brindado información esencial sobre la variación espacial y temporal de las cubiertas vegetales, contribuyendo a la estimación de importantes variables biofísicas y bioquímicas. Hasta el momento se han aplicado con éxito enfoques empíricos basados en índices de vegetación que han obtenido un nivel de precisión satisfactorio para estimar importantes parámetros biofísicos de la vegetación como el contenido en clorofila (Blackburn 1998; Broge y Mortensen 2002) y nitrógeno (Hansen y Schjoerring 2003; Martin et al. 2008). Otras aproximaciones basadas en el uso de modelos físicos de transferencia radiactiva han demostrado igualmente su utilidad para la estimación de variables de 
interés como el contenido de agua de la vegetación (Trombetti et al. 2008; Yebra y Chuvieco 2009).

En este contexto se inscriben las actividades del proyecto Biospec (http:// www.lineas.cchs.csic.es/biospec/), que explora el uso de la teledetección próxima y remota para estimar diversos parámetros biofísicos de la vegetación en una zona de dehesa localizada al Noreste de la provincia de Cáceres (España). Para ello se ha realizado una intensa campaña de campo que ha permitido recopilar un conjunto muy importante de datos sobre el estado de la vegetación y sobre su comportamiento espectral, los cuales están siendo utilizados para calibrar y validar modelos empíricos y físicos para la estimación de variables biofísicas a partir de datos de teledetección obtenidos a diversas escalas espaciales y temporales. Debido a la similitud entre los ecosistemas estudiados, se pretende extrapolar los resultados obtenidos con las mediciones de campo realizados en la zona de Cáceres (España) y aplicarlos sobre la zona de estudio propuesta en el presente estudio.

En las últimas décadas la teledetección ha sido utilizada ampliamente para la estimación de biomasa en pasto (Cho et al. 2007; Edirisinghe et al. 2012; Kogan et al. 2004; Malmstrom et al. 2009; Todd et al. 1998). A escala continental, sensores de baja resolución espacial, tales como NOAA AVHRR (Kogan et al. 2004) y MODIS (Muukkonen y Heiskanen 2007), han sido muy utilizados debido a su adecuada cobertura espacial y frecuencia de adquisición. Sin embargo, para cuantificar biomasa a escalas locales, es necesario contar con información obtenida por sensores con una resolución espacial más fina, como la obtenida por Landsat TM y ETM+ (Malmstrom et al. 2009; Meng et al. 2007; Todd et al. 1998). En este caso, el enfoque más frecuente consiste en relacionar índices de vegetación con datos de biomasa tomados en campo a través de modelos estadísticos (Cho et al. 2007; Edirisinghe et al. 2012; Malmstrom et al. 2009; Meng et al. 2007; Muukkonen y Heiskanen 2007; Todd et al. 1998).

Para la estimación de los stocks de carbono a partir de la biomasa, la Organización de Naciones Unidas para la Agricultura y la Alimentación (FAO) propone aplicar una fracción de carbono (Marklund y Schoene 2006) cuyo valor varía entre el $45 \%$ y $53 \%$, utilizándose por defecto, generalmente, un valor de $50 \%$. Cuando se tiene información de la especie sobre la que se quiere estimar el contenido de carbono se pueden aplicar fracciones más acordes a las mismas (García et al. 2010). En el departamento de Olancho, la ganadería, es de los rubros de mayor importancia económica y social, tanto por su aporte a la alimentación de la población como por su contribución en la generación de divisas y empleo en el área rural y en el campo de la agroindustria. Existen en el país 86,829 explotaciones dedicadas a la 
crianza de ganado bovino, de las cuales un $21.5 \%(18,648)$ existen en Olancho y de estas más del $50 \%$ son menores de 5 has, el inventario ganadero bovino del departamento es de 453,294 cabezas, que representan $24.4 \%$ del total nacional (Quiel y Pineda 2010).

La producción de leche es mayor en invierno, con una producción diaria de leche de 452,539 litros, (con una producción promedio de 4.3 litros), con un valor aproximado de 1.33 millones de lempiras, en comparación con la producción del verano que es de 264,830 litros (con una producción promedio de 3.5 litros), con un valor de 1.23 millones de Lempiras.

La actividad ganadera y lechera se concentra especialmente en el Valle del Guayape, este valle tiene una superficie de 93,000 has, cuenta con un total de 10,108 fincas de doble propósito, que producen cerca del $70 \%$ de la producción del departamento y un $12 \%$ de la producción nacional. Existen alrededor de 49 plantas industriales procesadoras de leche, que producen aproximadamente 40,000 libras diarias de lácteos, entre ellos quesillo, queso en sus diferentes modalidades y mantequilla, como resultado del procesamiento de 112, 270 litros de leche por día, generando un valor de 264.7 millones de Lempiras anualmente (Quiel y Pineda 2010).

El ganado bovino está ligado a la industria de transformación de sus productos. En relación a la industria de lácteos, se comercializan tanto en los principales plazas del país como en el mercado salvadoreño, en cuanto a la carne también se vende en el mercado local e internacional, para citar un ejemplo, existe una empacadora de carne denominada C\&D localizada en Catacamas, que comercializa sus productos a nivel local, en las grandes plazas de mercados del país y en mercados externos principalmente a Estados Unidos y Puerto Rico.

La Ganadería es un rubro muy importante para la generación de ingresos en los municipios de: Catacamas, Juticalpa, Culmí, San Esteban, Gualaco, San Francisco de la Paz, San Francisco de Becerra, Salamá y Guayape.

\section{Área de Estudio}

El área de estudio abarca las zonas de pastos del Departamento de Olancho (Oriente de Honduras). 
La climatología de la Región de Olancho es variada, predominando las variantes lluvioso de altura (25.8\%) y muy lluvioso de transición (24.2 \%). De acuerdo con la clasificación climática elaborada por el meteorólogo hondureño Edgardo Zúniga Andrade, la provincia climática "lluvioso de altura" se caracteriza por presentar un período lluvioso de seis meses siendo los más lluviosos junio y septiembre y los más secos febrero y marzo. La humedad relativa es de 72 a $74 \%$ con temperaturas de $21^{\circ} \mathrm{C}$ y $10^{\circ} \mathrm{C}$. Por su parte, en la provincia "muy lluvioso de transición" los meses más lluviosos son junio y octubre y los menos lluviosos marzo y abril. La precipitación promedio anual es de 250 a $1200 \mathrm{~mm}$ con una humedad relativa de 86\% (ANED 2013).

Las precipitaciones son muy variadas, el rango extremo de precipitaciones oscila entre $900 \mathrm{~mm}$ en los valles occidentales y los $3000 \mathrm{~mm}$ de las áreas más orientales. La diferencia estriba en que los primeros están situados en las cuencas intramontañosas a sotavento de los vientos húmedos del este. Por el contrario, las planicies orientales, pertenecientes a las cuencas de los ríos Patuca, Coco o Segovia, están abiertas a la influencia directa del mar Caribe debido a su cercanía y a la ausencia de relieves montañosos apreciables. No obstante el intervalo más común de precipitaciones está comprendido entre los 1300 y los $1700 \mathrm{~mm}$, distribuidos en 8 a 10 meses, siendo Marzo y Abril los meses más secos (ANED 2013).

Las temperaturas medias progresan paulatinamente de oeste a este de la Región Valles de Olancho, desde los $23,4^{\circ} \mathrm{C}$ hasta alcanzar los $27^{\circ} \mathrm{C}$. Las zonas más calientes son las áreas del valle de Azacualpa y Patuca. Las zonas más frías corresponden con las áreas montañosas de Agalta, y sierra de Patuca. El descenso estacional es de pocos grados pero suficientes para generar brumas y nieblas en áreas elevadas y para generar bosques nublados en los principales sistemas montañosos (ANED 2013).

La evapotranspiración sigue un patrón inverso a las temperaturas anuales, las zonas montañosas, especialmente en la Sierra Agalta, tienen la evapotranspiración más baja, con valores inferiores a los $1100 \mathrm{~mm}$ anuales, mientras que las cuencas bajas de los ríos Patuca y Segovia en la región Biosfera, tienen los valores más altos, con más de $1800 \mathrm{~mm}$ anuales. Salvo en áreas orientales muy localizadas, la evapotranspiración en los meses secos supera claramente los valores de precipitación, ocasionando un déficit hídrico estacional. La vegetación en este caso también presenta una estacionalidad en sus formas de vida con una marcada diferenciación fenológica (floración y fructificación en los meses de verano) (ANED 2013). 
El relieve de la Región Valles de Olancho se caracteriza por presentar áreas planas asociadas a valles fluviales de relativa poca altitud, con pendientes menores del 3\%, así como zonas onduladas ( 3 a $60 \%$ ) y escarpadas (mayores del $60 \%$ ). Las áreas con mayor pendiente se encuentran en la franja norte de la región, específicamente en la sierra de Agalta, mientras que las zonas onduladas se ubican en su mayoría hacia el oriente de la región (ANED 2013).

La altitud de la Región de Olancho oscila entre los 100 msnm de las partes más bajas de las cuencas de los ríos Coco y Patuca, en el extremo oriental del municipio de Catacamas hasta los 2590 msnm de las cumbres de la sierra de Agalta. Se puede observar un promedio de altura por municipio decreciendo desde Guaimaca hasta Catacamas en su parte sur y central (penetrando en el Valle de Olancho) y creciendo según el mismo eje en la franja norte (Sierra de Agalta). A pesar de su relieve montañoso, no se encuentran mesetas ni altiplanos excepto en la parte central de Guaimaca (ANED 2013).

\section{Resultados}

Una vez analizada la información del Sistema Nacional de Información Territorial (SINIT), y aplicados los procesos correspondientes para la identificación del área de estudio, se obtuvo una cobertura de pastos para el Departamento de Olancho de $670,749.79$ ha lo que representa aproximadamente un $28 \%$ de la extensión total del Departamento.

La composición del mapa de la zona de estudio se presenta en la Figura 2.

Basado en la experiencia personal y en algunos procedimientos utilizados por otros laboratorios se elaboró el Protocolo de Radiometría de Campo del Laboratorio de Radiometría del Departamento de Ciencia y Tecnologías de la Información Geográfica - FACES/UNAH, el cual, debido a su contenido, se presenta como anexo a este artículo. 


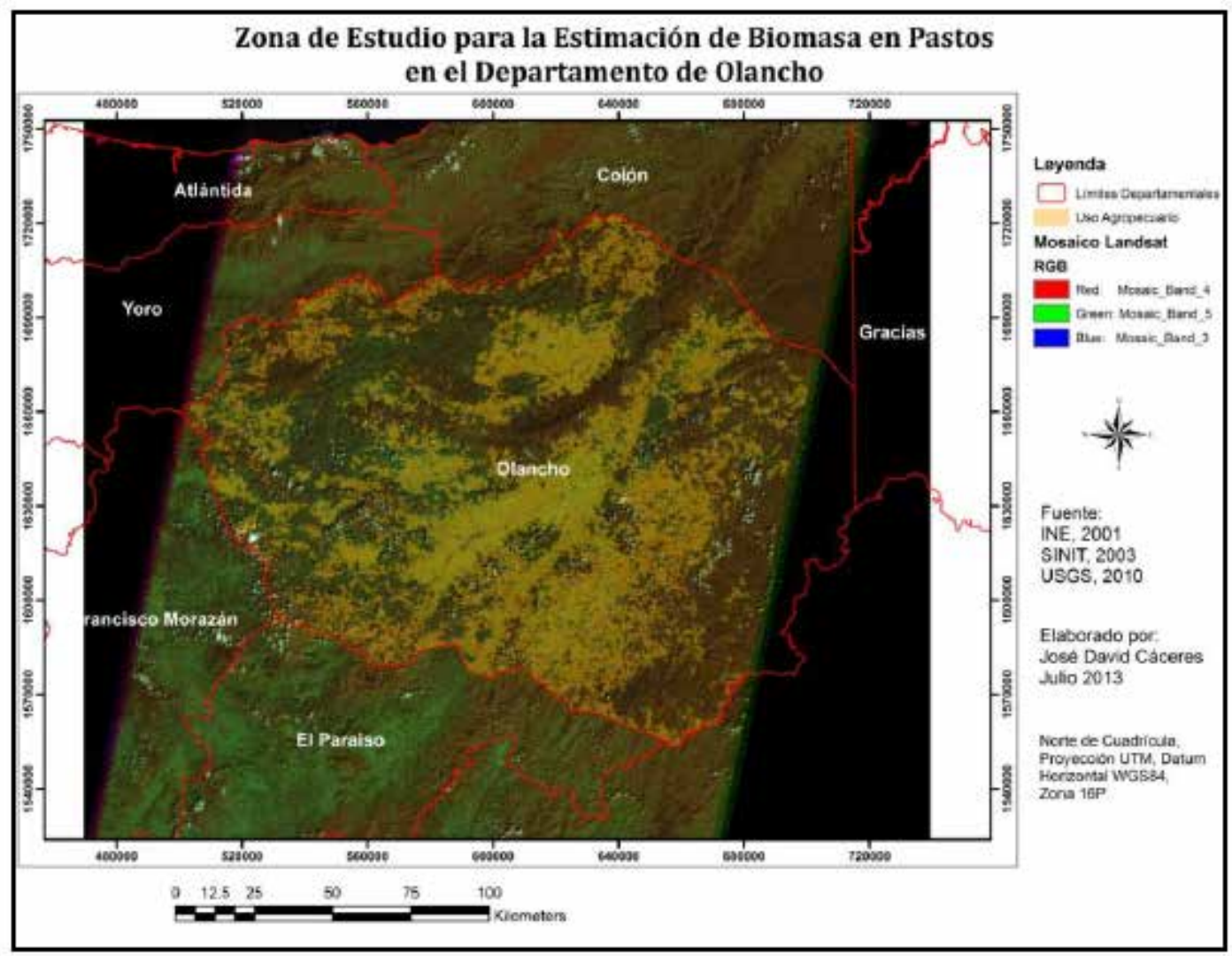

Figura 2. Zona de Área de Estudio

\section{Conclusiones}

- El contar con un protocolo de medición de radiometría de campo permitirá que los procedimientos para la toma de datos con el equipo que posee el Laboratorio de Radiometría del Departamento de Ciencia y Tecnologías de la Información Geográfica sean estandarizados, lo que proporcionará una mejor comparación entre los modelos generados a partir de estas muestras.

- La cobertura de pastos en el Departamento de Olancho cubre una parte significativa del territorio (aproximadamente $28 \%$ de su superficie) lo cual permite obtener un panorama general y representativo del comportamiento de los pastos a nivel nacional. 
- La información territorial con la que dispone el Sistema Nacional de Información Territorial (SINIT) constituye un insumo fundamental para el análisis de coberturas y para la gestión del territorio en general.

\section{Agradecimientos}

Al Sistema Nacional de Información Territorial (SINIT) por la disponibilidad de la información espacial del territorio hondureño. Al Servicio Geológico de los Estados Unidos (USGS) por la disponibilidad gratuita de las imágenes Landsat.

\section{Bibliografía}

- ANED (2013). Plan de Desarrollo Regional con Enfoque de Ordenamiento Territorial de la Región Valles de Olancho. In SEPLAN (Ed.). Honduras

- Blackburn, G.A. (1998). Quantifying chlorophylls and carotenoids at leaf and canopy scales; an evaluation of some hyperspectral approaches. Remote Sensing of Environment, 6, 273-285

- Broge , N.H., \& Mortensen, J.V. (2002). Deriving green crop area index and canopy chlorophyll density of winter wheat from spectral reflectance data. Remote Sensing of Environment, 81, 45-57

- Cho, M.A., Skidmore, A., Corsi, F., van Wieren, S.E., \& Sobhan, I. (2007). Estimation of green grass/herb biomass from airborne hyperspectral imagery using spectral indices and partial least squares regression. International Journal of Applied Earth Observation and Geoinformation, 9, 414-424

- Edirisinghe, A., Clark, D., \& Waugh, D. (2012). Spatio-temporal modelling of biomass of intensively grazed perennial dairy pastures using multispectral remote sensing. International Journal of Applied Earth Observation and Geoinformation, 16, 5-16

- García, M., Riaño, D., Chuvieco, E., \& Danson, F.M. (2010). Estimating biomass carbon stocks for a Mediterranean forest in central Spain using LiDAR height and intensity data. Remote Sensing of Environment, 114, 816-830 
- Hansen, P.M., \& Schjoerring, J.K. (2003). Reflectance measurement of canopy biomass and nitrogen status in wheat crops using normalized difference vegetation indices and partial least squares regression. Remote Sensing of Environment, 86, 542-553

- Kogan, F., Stark, R., Gitelson, A., Jargalsaikhan, L., Dugrajav, C., \& Tsooj, S. (2004). Derivation of pasture biomass in Mongolia from AVHRR-based vegetation health indices. International Journal of Remote Sensing, 25, 2889-2896

- Malmstrom, C.M., Butterfield, H.S., Barber, C., Dieter, B., Harrison, R., Qi, J., Riaño, D., Schrotenboer, A., Stone, S., Stoner, C.J., \& Wirka, J. (2009). Using Remote Sensing to Evaluate the Influence of Grassland Restoration Activities on Ecosystem Forage Provisioning Services. Restoration Ecology, 17, 526-538

- Marklund, L.G., \& Schoene, D. (2006). Global assessment of growing stock, biomass and carbon stock. In FAO (Ed.), Forest resources assessment programme 2005. Working paper 106. Rome (Italy): Food and Agriculture Organization of the United Nations (FAO)

- Martin, M.E., Plourde, L.C., Ollinger, S.V., Smith, M.L., \& McNeil, B. (2008). A generalizable method for remote sensing of canopy nitrogen across a wide range of forest ecosystems. Remote Sensing of Environment, 12, 3511-3519

- Meng, Q., Cieszewski, C.J., Madden, M., \& Borders, B. (2007). A linear mixedeffects model of biomass and volume of trees using Landsat ETM+ images. Forest Ecology and Management, 244, 93-101

- Muukkonen, P., \& Heiskanen, J. (2007). Biomass estimation over a large area based on standwise forest inventory data and ASTER and MODIS satellite data: A possibility to verify carbon inventories. Remote Sensing of Environment, 107, 617-624

- Quiel, P., \& Pineda, G. (2010). Dinámicas territoriales en Olancho entre los recursos naturales y la agricultura. In. Honduras: Rimisp - Centro Latinoamericano para el Desarrollo Rural

- Todd, S.W., Hoffer, R.M., \& Milchunas, D.G. (1998). Biomass estimation on grazed and ungrazed rangelands using spectral indices. International Journal of Remote Sensing, 19, 427-438 
- Trombetti, M., Riaño, D., Rubio, M.A., Cheng, Y.B., \& Ustin, S.L. (2008). Multitemporal vegetation canopy water content retrieval using Artificial Neural Networks for the USA. Remote Sensing of Environment, 112, 203-215

- Yebra, M., \& Chuvieco, E. (2009). Linking ecological information and radiative transfer models to estimate fuel moisture content in the Mediterranean region of Spain : Solving the ill-posed inverse problem. Remote Sensing of Environment, $113,2403-2411$ 